\title{
Microcrystal electron diffraction of the peptide Gramicidin D
}

\author{
Nicole Hoefer ${ }^{1}$ and David McComb ${ }^{2}$
}

1. Center for Electron Microscopy and Analysis, The Ohio State University, Columbus, Ohio, United States, ${ }^{2}$ Center for Electron Microscopy and Analysis, The Ohio State University, Columbus, OH, USA, COLUMBUS, Ohio, United States

Microcrystal electron diffraction (micro-ED) is a cryo-TEM technique that can be used to determine the atomic structure of proteins, peptides, and small molecules [1]. Micro-ED data is obtained by continuously tilting the crystal in the cryo-TEM while recording diffraction information [2]. This method has been successfully used for the structure determination of proteins such as proteinase K, lysozyme, adenosine A2A receptor (G-protein coupled receptor) and peptides such as an Alzheimer associated amyloid- $\beta$ (2034), SVQIVY (tau protein fragment) and others [3-7].

Growing diffraction quality crystals of proteins and peptides can be challenging. X-ray diffraction techniques typically require crystals that are at least $1 \mu \mathrm{m}$ in each direction. Crystals smaller than that could not be utilized in single crystal diffraction studies until the advent of micro-ED. In this technique the ideal thickness of the crystal perpendicular to the incident beam is in the nanometer range [2].

In this contribution we report on our research to determine the structure of gramicidin $\mathrm{D}$, a peptide antibiotic produced non-ribosomally by Bacillus brevis [8]. It acts, in part, by creating pores in membranes, rendering them incapable of supporting life-sustaining transmembranal gradients. Gramicidin is a highly apolar pentadecapeptide consisting of alternating D- and L-amino acids. Naturally occurring gramicidin is a mixture of isoforms: $\mathrm{gA}(80 \%), \mathrm{gB}(6 \%)$, and $\mathrm{gC}(14 \%)$. The amino acid sequence of $\mathrm{gA}$ is:

Formyl-NH-L-Val-Gly-L-Ala-D-Leu-L-Ala-D-Val-L-Val-D-Val-L-Trp-D-Leu-L-Trp11-DLeu-L-Trp-D-Leu-L-Trp-CO-NH-CH2-CH2-OH.

In $\mathrm{gB}$ and $\mathrm{gC}$, Trp at position 11 is replaced by L-Phe and L-Tyr, respectively. The ion conducting form of $\mathrm{gD}$ is generally considered to be a dimer. Gramicidin exists in two major conformations; a head-tohead, single stranded helical dimer and a left- or right-handed intertwined, parallel or antiparallel, double stranded double helix [9].

The peptide was dissolved in a mixture of ethanol / PEG 4000 and crystallized in batch-mode at $4^{\circ} \mathrm{C}$. Small plate-like crystals formed. The crystals in the presence of ethanol / PEG 4000 mixture were transferred onto Quantifoil R 2/2 grids and access solvent was removed by vacuum suction. The grids were flash frozen in liquid nitrogen and transferred into a Glacios cryo-TEM equipped with a Ceta-D camera. Datasets from several crystals were successfully collected. We will discuss the data processing in XDS and our progress on solving and refining the structure.

The authors acknowledge funding from the Foundation For A Better World.

\section{References}

[1] Nannenga, BL \& Gonen, T, Nat. Methods 16, 369-379 (2019).

[2] Shi D, et al., Nat Protoc. 2016 May;11(5):895-904.

[3] Nannenga BL, et al., Nat Methods. 2014 Sep;11(9):927-930.

[4] Martynowycz MW, et al., Structure. 2019 Oct 1;27(10):1594-1600.e2.

[5] Martynowycz, MW, et al., bioRxiv 2020.09.27.316109 (2020) 
[6] Warmack RA, et al., Nat Commun. 2019 Jul 26;10(1):3357

[7] Seidler PM, et al., J Biol Chem. 2019 Nov 1;294(44):16451-16464.

[8] Wallace BA, Annu Rev Biophys Biophys Chem. 1990;19:127-57.

[9] Höfer N, et al., Cryst Growth Des. 2011 Apr 6;11(4):1182-1192. 